Published in "Diagnostic Microbiology and Infectious Disease

83(3): 234-236, 2015"

which should be cited to refer to this work.

\title{
CHROMagar Acinetobacter medium for detection of carbapenemase-producing Acinetobacter spp. strains from spiked stools
}

\author{
Delphine Girlich ${ }^{\mathrm{a}, *}$, Patrice Nordmann ${ }^{\mathrm{b}, \mathrm{c}}$ \\ a Faculté de Médecine et Université Paris Sud, K.-Bicêtre, France \\ ${ }^{\mathrm{b}}$ Emerging Antibiotic Resistance Unit, Medical Molecular Microbiology, Department of Medicine, Faculty of Science, University of Fribourg, Fribourg, Switzerland \\ ${ }^{\mathrm{c}}$ HFR-Hôpital Cantonal, Fribourg, Switzerland
}

\begin{abstract}
The recently modified CHROMagar Acinetobacter medium was evaluated for detection of carbapenemase-producing Acinetobacter baumannii from spiked stools. A total of 45 Acinetobacter spp. isolates were tested. The CHROMagar Acinetobacter medium had a high sensitivity of $86.5 \%$ and a specificity of $75 \%$. This medium is likely to be most useful for controlling outbreaks and in endemic situations.
\end{abstract}

Acinetobacter spp. are an important source of healthcare-associated infections. The spread of carbapenemase-producing Acinetobacter spp. is increasingly reported worldwide associated with multidrug resistance (Bonnin et al., 2013a; Poirel and Nordmann, 2006; Wareham et al., 2008). Therefore, targeted surveillance of high-risk patients based on screening of carriers is essential to control the spread of carbapenem-resistant Acinetobacter spp. (CRAB). Resistance to carbapenems in Acinetobacter spp. may result from decreased permeability of the outer membrane, modification of penicillin-binding proteins, production of carbapenemases, and mostly from combined resistance mechanisms (Bonnin et al., 2013a; Poirel and Nordmann, 2006; Fernández-Cuenca et al., 2003). Carbapenemases in Acinetobacter spp. belong to Ambler class A (KPC and some GES variants) (Bonnin et al., 2013b; Robledo et al., 2010), class B (VIM, IMP, SIM, NDM) (Bonnin et al., 2012; Poirel and Nordmann, 2006), and mostly class D (oxacillinases) (Bonnin et al., 2013a). Five main groups of oxacillinases with carbapenemase activity have been identified in A. baumannii, i.e., the intrinsic chromosomal OXA-51-like and the acquired chromosomally and plasmid-encoded OXA-23-like, OXA-40-like, OXA-58-like, and OXA-143-like enzymes (Bonnin et al., 2013a; Higgins et al., 2009; Poirel and Nordmann, 2006). When the bla OXA-51-like genes are expressed at a basal level, they do not confer carbapenem resistance. However, the insertion of the insertion sequence ISAba1 upstream of

\footnotetext{
* Corresponding author. Tel.:+33-1-45212019; fax: +33-1-45216340.

E-mail address: dgirlich@yahoo.fr (D. Girlich).
}

the $b l a_{\mathrm{OXA}-51-l i k e}$ gene may lead to overexpression of this oxacillinase gene leading to carbapenem resistance (Turton et al., 2006). Selective media developed for detection of carbapenem-resistant Enterobacteriaceae are inappropriate for detection of CRAB, since Acinetobacter spp. are intrinsically resistant to several carbapenem molecules that are contained in those selective media. CHROMagar Acinetobacter (CHROMagar, Paris, France) is a selective medium designed for rapid identification of CRAB inhibiting the growth of yeast, carbapenemsusceptible Gram negatives and Gram positives, and coloring the colonies of Acinetobacter spp. in red since it contains a chromogenic molecule (Ajao et al., 2011; Gordon and Wareham, 2009). A new formula of this medium has been recently developed (Song et al., 2013). As compared to the previous version of the CHROMagar Acinetobacter medium, it contains an antimicrobial selective supplement (CR102; CHROMagar) aimed to select for CRAB.

While previous studies on previous formulas of this medium included strains for which the molecular mechanisms of resistance was not determined, our aim was to evaluate the performance of this CHROMagar Acinetobacter medium using a set of precisely moleculardefined carbapenemase producers. Spiked stools were used to mimic the in vivo colonization of stools.

Forty-five Acinetobacter spp. isolates (mostly A. baumannii) were tested, including 37 acquired carbapenemase producers as follows: Ambler class A (GES-11-, GES-14-type) producers $(n=2)$, Ambler class B (IMP, VIM, SIM, NDM-type) producers $(n=7)$, Ambler class D (OXA-23-type, OXA-40-type, OXA-58-type) producers $(\mathrm{n}=28)$, and 8 
strains that do not produce acquired carbapenemases $(n=8)$, the last being either carbapenem resistant or carbapenem susceptible. OXA-23 producers are by far the most widespread carbapenemase producers in A. baumannii (Bonnin et al., 2013a). MIC values of imipenem and meropenem ( $A$. baumannii is naturally resistant to ertapenem) were determined by E-test and interpreted according to the CLSI guidelines updated in 2014. MIC breakpoints for imipenem and meropenem against $A$. baumannii were susceptibility for MICs $\leq 2 \mu \mathrm{g} / \mathrm{mL}$, intermediate resistance $4 \mu \mathrm{g} / \mathrm{mL}$, and resistance for MICs $\geq 8 \mu \mathrm{g} / \mathrm{mL}$, as updated in CLSI (2014). The CHROMagar Acinetobacter medium was prepared as recommended by the manufacturer from dehydrated powder and liquid supplement added in the form of antimicrobial selective supplement (CR102). Bacterial suspensions of the strains with an optical density of $0.5 \mathrm{McFarland}$ (inoculum of $\sim 5 \times 10^{7} \mathrm{CFU} / \mathrm{mL}$ ) were serially diluted in water. Ten fold dilutions were made. To quantify the viable bacteria in each dilution, trypticase soy agar was inoculated concomitantly with $100 \mu \mathrm{L}$ of suspension and incubated overnight at $37^{\circ} \mathrm{C}$; the number of viable colonies was counted the following day. Spiked fecal samples were made by adding $100 \mu \mathrm{L}$ of each dilution to $900 \mu \mathrm{L}$ of fecal suspension that was obtained by suspending $4 \mathrm{~g}$ of freshly pooled feces from four healthy volunteers in $40 \mathrm{~mL}$ of distilled water, as previously described (Naas et al., 2011). A fecal suspension without the addition of a bacterial strain was used as negative control. The lowest detection limit of the carbapenemase producers was determined by plating $100 \mu \mathrm{L}$ of each dilution on CHROMagar Acinetobacter medium (CHROMagar). Viable bacteria were counted after $24 \mathrm{~h}$ of culture at $37^{\circ} \mathrm{C}$. The sensitivity and specificity were determined using a cutoff value set at $\geq 1 \times 10^{3} \mathrm{CFU} / \mathrm{mL}$, as previously described (Nordmann

Table 1

Sensitivity of detection of the CHROMagar Acinetobacter medium from spiked fecal samples.

\begin{tabular}{|c|c|c|c|c|}
\hline & $\beta$-Lactamase content & IPM MIC $(\mu \mathrm{g} / \mathrm{mL})$ & MEM MIC $(\mu \mathrm{g} / \mathrm{mL})$ & Lowest detection limit $(\mathrm{CFU} / \mathrm{mL})^{\mathrm{a}}$ \\
\hline \multicolumn{5}{|l|}{ Carbapenemase Ambler class A } \\
\hline A. baumannii KOW & GES-11 & 6 & 8 & $1 \times 10^{1}$ \\
\hline A. baumannii RB & GES-14 & 32 & 32 & $1 \times 10^{1}$ \\
\hline \multicolumn{5}{|l|}{ Carbapenemase Ambler class B } \\
\hline A. baumannii IMP & IMP-1 & 4 & 6 & $1 \times 10^{1}$ \\
\hline A. baumannii IMP4 & IMP-4 & 24 & 16 & $1 \times 10^{2}$ \\
\hline Acinetobacter genomospecies 16 & VIM-4 & $>32$ & $>32$ & $1 \times 10^{2}$ \\
\hline A. baumannii SIM & SIM-1 & $>32$ & $>32$ & $1 \times 10^{1}$ \\
\hline A. baumannii SLO & NDM-1 & $>32$ & $>32$ & $1 \times 10^{1}$ \\
\hline A. baumannii ALG & NDM-1 & $>32$ & $>32$ & $1 \times 10^{1}$ \\
\hline A. baumannii EGY & NDM-2 & $>32$ & $>32$ & $1 \times 10^{1}$ \\
\hline \multicolumn{5}{|l|}{ Carbapenemase Ambler class D } \\
\hline A. baumannii 23-B2 & OXA-23 & $>32$ & $>32$ & $1 \times 10^{1}$ \\
\hline A. baumannii 23-C2 & OXA-23 & $>32$ & $>32$ & $1 \times 10^{1}$ \\
\hline A. baumannii 23-D2 & OXA-23 & $>32$ & $>32$ & $1 \times 10^{1}$ \\
\hline A. baumannii 23-E2 & OXA-23 & $>32$ & $>32$ & $1 \times 10^{1}$ \\
\hline A. baumannii 23-F2 & OXA-23 + PER-1 & $>32$ & $>32$ & $1 \times 10^{1}$ \\
\hline A. baumannii $23-\mathrm{G} 2$ & OXA-23 & $>32$ & $>32$ & $1 \times 10^{1}$ \\
\hline A. baumannii 23-G4 & OXA-23 & $>32$ & $>32$ & $1 \times 10^{2}$ \\
\hline A. baumannii $26-\mathrm{C} 2$ & OXA-26 & $>32$ & $>32$ & $1 \times 10^{1}$ \\
\hline A. baumannii 40-A1 & OXA-40 & $>32$ & $>32$ & $1 \times 10^{1}$ \\
\hline A. baumannii $40-\mathrm{A} 2$ & OXA-40 & $>32$ & $>32$ & $2 \times 10^{1}$ \\
\hline A. baumannii 40-A3 & OXA-40 & $>32$ & $>32$ & $2 \times 10^{1}$ \\
\hline A. baumannii 40-A4 & OXA-40 & $>32$ & $>32$ & $1 \times 10^{1}$ \\
\hline A. baumannii 40-A5 & OXA-40 & $>32$ & $>32$ & $1 \times 10^{1}$ \\
\hline A. baumannii 40-D7 & OXA-40 & $>32$ & $>32$ & $1 \times 10^{1}$ \\
\hline A. baumannii 40-D8 & OXA-40 & $>32$ & $>32$ & $1 \times 10^{1}$ \\
\hline A. baumannii 40-D9 & OXA-40 & $>32$ & $>32$ & $\underline{1 \times 10^{3}}$ \\
\hline A. baumannii 72-D5 & OXA-72 & $>32$ & $>32$ & $\overline{1 \times 10^{1}}$ \\
\hline A. baumannii 58-A2 & OXA-58 & $>32$ & $>32$ & $2 \times 10^{2}$ \\
\hline A. baumannii 58-A4 & OXA-58 & $>32$ & 24 & $2 \times 10^{2}$ \\
\hline A. baumannii 58-A7 & OXA-58 + PER-2 & $>32$ & 12 & $1 \times 10^{1}$ \\
\hline A. baumannii 58-B1 & OXA-58 & 24 & 32 & $1 \times 10^{1}$ \\
\hline A. baumannii 58-B2 & OXA-58 & $>32$ & 32 & $2 \times 10^{2}$ \\
\hline A. baumannii SWE & OXA-58 & $>32$ & 24 & $5 \times 10^{3}$ \\
\hline A. baumannii BAR & OXA-58 & 4 & 1 & $2 \times 10^{6}$ \\
\hline A. baumannii ITA & OXA-58 & $>32$ & 16 & $6 \times 10^{2}$ \\
\hline A. baumannii GRE & OXA-58 & $>32$ & $>32$ & $1 \times 10^{3}$ \\
\hline A. haemolyticus 58-A10 & OXA-58 & $>32$ & 8 & $>\overline{1 \times 10^{6}}$ \\
\hline \multicolumn{5}{|l|}{ Multiple carbapenemases } \\
\hline A. baumannii LIB & GES-11 + OXA-23 & $>32$ & $>32$ & $1 \times 10^{1}$ \\
\hline \multicolumn{5}{|l|}{ No acquired carbapenemase } \\
\hline A. baumannii $\mathrm{CB} 3$ & None & 1 & 0.25 & $>1 \times 10^{\underline{6}}$ \\
\hline A. baumannii CB4 & None & 0.25 & 0.25 & $>\overline{1 \times 10^{6}}$ \\
\hline A. baumannii $\mathrm{CB} 6$ & None & 0.25 & 0.12 & $>\overline{1 \times 10^{6}}$ \\
\hline A. baumannii CA9 & RTG-4 & 0.38 & 0.25 & $>\overline{1 \times 10^{6}}$ \\
\hline A. baumannii CA1 & GES-12 + OXA-51 + ISAba1 & 32 & 32 & $\overline{1 \times 10^{2}}$ \\
\hline A. baumannii CA3 & OXA-51 + ISAba 1 & 3 & 3 & $1 \times 10^{2}$ \\
\hline A. baumannii CA6 & SHV-5 & 6 & 8 & $4 \times 10^{4}$ \\
\hline A. baumannii CA7 & PER-1 & 1.5 & 0.75 & $>\overline{1 \times 10^{6}}$ \\
\hline
\end{tabular}

Abbreviations: IPM = imipenem; MEM = meropenem.

Underlined CFU counts are considered as negative results (cutoff values set at $\geq 1 \times 10^{3} \mathrm{CFU} / \mathrm{mL}$ ).

a One milliliter of stools contains $100 \mathrm{mg}$ of stool. 
et al., 2012), i.e., a limit detection value of $1 \times 10^{3} \mathrm{CFU} / \mathrm{mL}$ or higher was considered as lack of detection (Table 1 ). This value may correspond to a low-level carriage of multidrug-resistant bacteria in stools.

Carbapenemase-producing A. baumannii were well detected from spiked stools except some OXA-58 producers (Table 1). Lack of detection was noticeable for 2 OXA-58 producers with high-level resistance to carbapenems. The sensitivity of detection of carbapenemaseproducing A. baumannii using the CHROMagar Acinetobacter medium (86.5\%) was lower than the sensitivity of detection of carbapenemresistant A. baumannii (91.7\%), similarly to what was previously reported for detection of multidrug-resistant $A$. baumannii in an outbreak situation in intensive care unit in 2009 in the UK (sensitivity of 91.7\%) (Gordon and Wareham, 2009). This difference may be due to a lower limit of detection that has been set here at $10^{3} \mathrm{CFU} / \mathrm{mL}$. Further studies should clinically validate this cutoff value with carbapenem-resistant $A$. baumannii isolates. Sensitivity would have been $94.6 \%$ when just considering growth or no growth, as done in this British study (Gordon and Wareham, 2009).

We identified specificity of the CHROMagar Acinetobacter medium of $75 \%$ that was lower than that previously reported by Gordon and Wareham (2009) (89.7\%). Our specificity result was lower since we included in "non-acquired carbapenemase-producers" 2 strains of $A$. baumannii with insertion of ISAba1 upstream of the naturally occurring bla $a_{\mathrm{OXA}-51}$ gene. This resulted in the overexpression of this oxacillinase gene and thus decreased susceptibility to carbapenems, as previously reported (Brown et al., 2005; Turton et al., 2006). Moran-Gilad et al. (2014) showed that CHROMagar Acinetobacter medium had a sensitivity of $100 \%$ for detection of isolates with MICs of imipenem $>32 \mu \mathrm{g} / \mathrm{mL}$ and a specificity of $100 \%$ for isolates with MICs of imipenem $<1 \mu \mathrm{g} / \mathrm{mL}$ (Moran-Gilad et al., 2014). Our study shows that this rule is not applicable in all cases (Table 1). Neither imipenem nor meropenem MIC could be strictly correlated with the detection limit of the CHROMagar Acinetobacter medium, as exemplified by the following strains, A. baumannii KOW, IMP, and CA6, which produce GES-11, IMP-1 and SHV-5, respectively. Although these strains showed similar MICs, the carbapenemase producers were specifically detected on the CHROMagar Acinetobacter medium (Table 1).

Noticeably, this medium showed a good specificity, since no other bacteria from stools were detected on the CHROMagar Acinetobacter medium. This result correlated with that of previous studies on previous formulations of this medium. Wareham and Gordon (2011) showed that the use of the KPC supplement enabled recovery of carbapenemresistant $A$. baumannii, distinguishable from carbapenem-resistant Enterobacteriaceae by the color of the colonies, and Barsoumian et al. (2013) showed that the CR102 supplement prevented the growth of other bacterial species even if carbapenem resistant (Barsoumian et al., 2013).

Overall, the studied screening medium has a good efficiency for detection of carriers of CRAB and is likely to be most useful during outbreaks or when CRAB is endemic. At least, based on this study performed with spiked stools, the CHROMagar Acinetobacter medium can detect not only Ambler class A (GES-type carbapenemase), Ambler class B, but also class Ambler D producers (in particular the most widespread OXA-23) (Mugnier et al., 2010). The CHROMagar Acinetobacter medium is well adapted for direct inoculation of patient samples (rectal swabs, stools, skin, and nasal samples) in any clinical settings, as recently shown by Song et al. (2013). Use of this screening medium based on detection of carbapenem resistance is of special interest since most of the carbapenem-resistant $A$. baumannii isolates express an acquired carbapenemase (here 36 out of 37 strains) and the carbapenem resistance trait is associated to multidrug resistance and vice versa.

\section{Funding}

This work was supported by a grant from the INSERM (U914) and from the Ministère de l'Education Nationale et de la Recherche, Université Paris XI, Paris, France.

\section{Transparency declarations}

None to declare.

\section{References}

Ajao AO, Robinson G, Lee MS, Ranke TD, Venezia RA, Harris AD, et al. Comparison of culture media for detection of Acinetobacter baumannii in surveillance cultures of critically-ill patients. Eur J Clin Microbiol Infect Dis 2011;30:1425-30.

Barsoumian A, Calvano T, Markelz AE, Cassiy R, Murray CK, Beckius ML, et al. Variations of CHROMagar Acinetobacter to detect imipenem-resistant Acinetobacter baumanniicalcoaceticus complex. Scand J Infect Dis 2013;45:446-52.

Bonnin RA, Poirel L, Naas T, Pirs M, Seme K, Schrenzel J et al. Dissemination of New Delhi metallo- $\beta$-lactamase-1-producing Acinetobacter baumannii in Europe. Clin Microbiol Infect 2012;18:E362-5.

Bonnin RA, Nordmann P, Nordmann P. Screening and deciphering antibiotic resistance in Acinetobacter baumannii: a state of the art. Exp Rev Anti Infect Ther 2013a;11:571-83.

Bonnin RA, Rotimi VO, Al Hubail M, Gasiorowski E, Al Sweih N, Nordmann P, et al. Wide dissemination of GES-type carbapenemases in Acinetobacter baumannii isolates in Kuwait. Antimicrob Agents Chemother 2013b;57:183-8.

Brown S, Young HK, Amyes SGB. Characterisation of OXA-51, a novel class D carbapenemase found in genetically unrelated clinical strains of Acinetobacter baumannii from Argentina. Clin Microbiol Infect 2005;11:15-23.

Clinical and Laboratory Standards Institute (CLSI). Performance standards for antimicrobial susceptibility testing: 24th informational supplement (M100-S24). Wayne, PA: CLSI; 2014.

Fernández-Cuenca F, Martínez-Martínez L, Conejo MC, Ayala JA, Perea EJ, Pascual A. Relationship between beta-lactamase production, outer membrane protein and penicillin-binding protein profiles on the activity of carbapenems against clinical isolates of Acinetobacter baumannii. J Antimicrob Chemother 2003;51:565-74.

Gordon NC, Wareham DW. Evaluation of CHROMagar Acinetobacter for detection of enteric carriage of multidrug-resistant Acinetobacter baumannii in samples from critically ill patients. J Clin Microbiol 2009;47:2249-51.

Higgins PG, Poirel L, Lehmann M, Nordmann P, Seifert H. OXA-143, a novel carbapenemhydrolyzing class D $\beta$-lactamase in Acinetobacter baumannii. Antimicrob Agents Chemother 2009;53:5035-8.

Moran-Gilad J, Adler A, Schwartz D, Navon-Venezia S, Carmeli Y. Laboratory evaluation of different agar media for isolation of carbapenem-resistant Acinetobacter spp. Eur J Clin Microbiol Infect Dis 2014;33:1909-13.

Mugnier PD, Poirel L, Naas T, Nordmann P. Worldwide dissemination of the bla $a_{\mathrm{OXA}-23}$ carbapenemase gene of Acinetobacter baumannii. Emerg Infect Dis 2010;16:35-40.

Naas T, Ergani A, Carrër A, Nordmann P. Real-time PCR for detection of NDM-1 carbapenemase genes from spiked stool samples. Antimicrob Agents Chemother 2011;55:4038-43.

Nordmann P, Girlich D, Poirel L. Detection of carbapenemase producers in Enterobacteriaceae by use of a novel screening medium. J Clin Microbiol 2012;50:2761-6.

Poirel L, Nordmann P. Carbapenem resistance in Acinetobacter baumannii: mechanisms and epidemiology. Clin Microbiol Infect 2006;12:826-36.

Robledo IE, Aquino EE, Santé MI, Santana JL, Otero DM, León CF, et al. Detection of KPC in Acinetobacter spp. in Puerto Rico. Antimicrob Agents Chemother 2010;54:1354-7.

Song W, Lee J, Lim TK, Park MJ, Kim HS, Kim JS. Modified CHROMagar Acinetobacter medium for direct detection of multi-drug resistant Acinetobacter strains in nasal rectal swab samples. Ann Lab Med 2013;33:193-5.

Turton JF, Ward ME, Woodford N, Kaufmann ME, Pike R, Livermore DM, et al. The role of ISAba1 in expression of OXA carbapenemase genes in Acinetobacter baumannii. FEMS Microbiol Lett 2006:258:72-7.

Wareham DW, Gordon NC. Modifications to CHROMagar Acinetobacter for improved selective growth of multi-drug resistant Acinetobacter baumannii. J Clin Pathol 2011 64:164-7.

Wareham DW, Bean DC, Khanna P, Hennessy EM, Krahe D, Ely A, et al. Bloodstream infection due to Acinetobacter spp: epidemiology, risk factors and impact of multidrug resistance. Eur J Clin Microbiol Infect Dis 2008;27:607-12. 